\title{
Qualidade de tomates de mesa cultivados em sistema orgânico e convencional no estado de Goiás
}

\author{
Abadia dos R Nascimento; Manoel S Soares Júnior; Márcio Caliari; Paulo M Fernandes; Janaína PM \\ Rodrigues; Webber T de Carvalho \\ UFG, Escola de Agronomia e Engenharia de Alimentos, C. Postal 131; 74690-900 Goiânia-GO; reyzynha@yahoo.com.br (autor \\ correspondente); mssoaresjr@hotmail.com; macaliari@ig.com.br; pmarcal@terra.com.br; janainapm@gmail.com; webbertavares@ \\ yahoo.com.br
}

\section{RESUMO}

A agricultura orgânica é um método de cultivo que visa o estabelecimento de sistemas agrícolas ecologicamente equilibrados e estáveis, economicamente produtivos e de elevada eficiência. O objetivo deste trabalho foi avaliar as características físicas, físico-químicas e sensoriais de cultivares de tomate de mesa cultivados em sistema orgânico e convencional. Dois experimentos foram realizados; no primeiro dez cultivares foram plantadas em sistema orgânico e os frutos em estádio de maturação 3 (quando apresentavam 60 a 90\% na coloração róseo-vermelha ou vermelha da casca) foram avaliados quanto às características físicas e sensoriais, e a polpa (mesocarpo) em relação às características físico-químicas. No segundo, a cultivar Débora produzida nos sistemas orgânico e convencional foi comparada quanto às mesmas características. Os maiores teores de sólidos solúveis (SS) foram observados nas cultivares Milenium, Andréia e Tathy 1, seguidas pelas cultivares Giovanna 1, Colibri, Débora, Setcopa e Bonus 1. Os menores valores de acidez total (AT) foram verificados nas cultivares Colibri, Andréa, Tathy 1, Débora e Setcopa, que não diferiram entre si $(\mathrm{p}>0,05)$ e também não diferiram das cultivares Bônus-1 e Stillus. As cultivares Andréa e Tathy 1 apresentaram as maiores relações SS/AT, não diferindo $(\mathrm{p}>0,05)$ das cultivares Débora, Setcopa e Colibri. A cultivar Débora, cultivada no sistema orgânico, foi a mais preferida em relação aos atributos textura (maciez), cor externa e interna, além de apresentar a menor acidez, maior relação SS/AT e densidade. Conclui-se que mais se destacaram ao nível das necessidades do produtor (maior massa e densidade) as cultivares Setcopa, Milenium, Giovanna 1, Stillus, Thaty 1 e Colibri, enquanto que para o consumidor, cuja necessidade esta mais voltada aos aspectos sensoriais e físico-químicos (cor, aroma, sabor, textura, sólidos solúveis e acidez), destacaram-se as cultivares Débora, Bônus-1, Andréa, Setcopa, Stillus, Thaty 1 e Colibri. A cultivar Débora obteve menor massa, volume e diâmetro longitudinal quando cultivada em sistema orgânico, assim como maior teor de sólidos solúveis e menor acidez, que refletiu em maior aceitação quanto ao aroma, sabor e textura pelos consumidores.

Palavras-chave: Solanum lycopersicon, cultivar, agricultura orgânica, análise físico-química, análise sensorial.

\begin{abstract}
Quality of tomatoes for fresh consumption grown in organic and conventional systems in the state of Goias, Brazil

Organic agriculture is a cultivation method that looks for the establishment of agricultural systems balanced and ecologically stable, economically productive and highly efficient. The objective of this work was to evaluate the physical, physico-chemical and sensory characteristics of tomato cultivars for fresh consumption grown on organic and conventional systems. Two experiments were carried out; on the first ten cultivars were grown under organic system and fruits at maturity stages 3 (when they presented 60 to $90 \%$ of pinkred color or red peel) were evaluated for physical, physicochemical and sensorial characteristics. On the second experiment, cultivar Deborah produced in organic and conventional systems was compared based on the same characteristics. The highest levels of soluble solids (SS) were observed in cultivars Milenium, Andréa and Tathy 1, followed by Giovanna 1, Colibri, Débora, Setcopa and Bonus 1 . The cultivars Colibri, Andréa, Thaty 1, Débora and Setcopa had the lowest values for total acidity (AT) and they did not differ ( $p>0.05)$, among themselves, neither from cultivars Bonus- 1 and Stillus. The cultivars Andréa and Tathy 1 had the highest ratio SS/TA and did not differ $(\mathrm{p}>0.05)$ from cultivars Débora, Setcopa and Colibri. Debora, when cultivated in the organic system was more preferred on attribute texture (tenderness), external and internal color, besides presenting a lower acidity, higher SS/TA ratio and density. It is concluded that the most outstanding, in terms of producer (greater mass and density) requirements, were the cultivars Setcopa, Milenium, Giovanna 1, Stillus, Thaty 1 and Colibri, while for the consumer, whose requirements are more focused on sensory and physico-chemical aspects (color, aroma, flavor, texture, soluble solids and acidity), cultivars Deborah, Bonus-1, Andrea, Setcopa, Stillus, Thaty 1 and Colibri stood out. Cultivar Deborah had lower mass, volume and longitudinal diameter when grown in organic system, as well as higher soluble solids and lower acidity, which resulted in greater acceptance for aroma, flavor and texture by consumers.
\end{abstract}

Keywords: Solanum lycopersicon, cultivar, organic agriculture, physicochemical analysis, sensory analysis.

\section{(Recebido para publicação em 28 de janeiro de 2013; aceito em 7 de outubro de 2013)} (Received on January 28, 2013; accepted on October 7, 2013)

\begin{abstract}
$\mathrm{A}$ tualmente, a qualidade de qualquer tipo de alimento (frescos ou processados) passou a ser considerada
\end{abstract}

fator de segurança alimentar e nutricional, sendo relacionada não só à sua produção em quantidade suficiente e acesso garantido, mas também à promoção do estado de saúde daqueles que o consomem. A composição bioquímica 
dos alimentos vem sendo alterada pelo sistema de produção, pois, a agricultura convencional lança mão de produtos químicos para fertilizar as plantas e para protegê-las contra pragas e doenças, comprometendo assim sua qualidade (Souza \& Resende, 2006).

$\mathrm{Na}$ produção de alimentos orgânicos, o agricultor não utiliza agrotóxicos e fertilizantes químicos de alta concentração e solubilidade e, sim, princípios e processos tecnológicos conservacionistas (Arbos et al., 2010). A agricultura orgânica vem ganhando cada vez mais reconhecimento social, político e científico em todo o mundo por estar fundamentada na aplicação de estratégias agroecológicas, mediante o uso de insumos locais, aumentando o valor agregado e propiciando uma cadeia de comercialização mais justa (Melo et al., 2009).

O mercado de produtos orgânicos é impulsionado pela demanda de consumidores preocupados com qualidade, saúde, questões ambientais e preservação. A produção orgânica apresenta melhor desempenho ambiental do que o sistema convencional, uma vez que melhora a conservação dos recursos naturais (Toledo et al., 2011).

O Brasil é um dos maiores produtores mundiais de tomate (Solanum lycopersicon), junto com China, Estados Unidos, Itália, Turquia, Espanha, Egito e México (Cardoso et al., 2010). Esta cultura encontra-se bastante desenvolvida no país e seu fruto está entre as hortaliças mais consumidas no mundo, globalmente enraizada no hábito alimentar. Em 2010, o Brasil se apresentou na nona posição mundial em produção (3.691.316 toneladas), décimo quarto colocado em área cultivada e o sexto em produtividade, com rendimento médio superior à média mundial (Faostat, 2012; Sanjinez-Argandona et al., 2011). O estado de Goiás foi responsável por $30,35 \%$ (1.120.135 toneladas) da produção nacional (IBGE, 2011).

O conceito de qualidade do tomate refere-se àqueles atributos que o consumidor e o produtor estimam que o produto deva possuir. Aos produtores, compete colher frutos de alto rendimen- to, com boas características sensoriais e propiciar eficiente armazenamento, enquanto os consumidores determinam sua qualidade pela aparência, textura, inexistência de deformidades e outros atributos sensoriais (Casquet, 1998). Além de não prejudicar a sua saúde, em relação à contaminação por microrganismos patogênicos e por resíduos químicos oriundos dos produtos utilizados durante a produção, transporte e armazenamento.

O tomate é uma das culturas nacionais de maior importância econômica, pois é a hortaliça mais industrializada na forma de inúmeros subprodutos, como extrato, polpa, pasta e, mais recentemente, o tomate seco.

Os tomates e seus derivados são ricos em compostos relacionados à saúde alimentar. Eles são boas fontes de carotenóides (em particular, licopeno), ácido ascórbico (vitamina $\mathrm{C}$ ), vitamina E, ácido fólico, flavonóides e potássio. Sendo que os principais antioxidantes do tomate são os carotenóides, ácido ascórbico e compostos fenólicos (Souza et al., 2008).

Atualmente, são escassas as informações relativas a cultivares de tomate de mesa adaptadas ao cultivo orgânico, como técnicas de manejo cultural, controle de pragas e doenças, manejo pós-colheita das diferentes variedades cultivadas e qualidade do tomate orgânico (Ferreira et al., 2010a; Melo et al., 2009). O interesse em estudar o tomate orgânico surgiu de evidências sobre uma mudança de hábito alimentar da população, na direção de uma crescente demanda de produtos orgânicos, a julgar pela presença desses produtos nas grandes redes de supermercados. Além disso, há ausência de informações sobre a qualidade das cultivares de tomate "de mesa" Andrea, Tathy 1, Bônnus 1 e Milenium, cultivadas no sistema orgânico, e falta de clareza em relação às possíveis diferenças físico-químicas e sensoriais existentes desses frutos quando produzidos em sistema convencional e orgânico.

Diante do exposto acima, objetivou-se avaliar as características físicas, químicas e sensoriais de dez cultivares de tomate de mesa cultivadas em siste- ma orgânico e comparar os atributos de qualidade dos frutos da cultivar Débora, quando produzidos nos sistemas orgânico e convencional.

\section{MATERIAL E MÉTODOS}

No primeiro experimento foram conduzidos em sistema orgânico dez cultivares de tomate "de mesa" (Giovanna 1, Colibri, Carmem, Andréa, Tathy 1, Milenium, Bônus-1, Stillus, Débora e Setcopa), na Fazenda Nossa Senhora Aparecida, situada no município de Hidrolândia, Goiás, durante a safra de 2009. O preparo do solo foi realizado por meio de duas passagens de grade aradora. $\mathrm{O}$ transplante das mudas foi realizado no dia 25 de julho e a colheita iniciou-se no final de outubro. Na adubação de plantio utilizou-se 200 $\mathrm{g}$ de esterco de minhoca e $50 \mathrm{~g}$ de termo fosfato Yorin por metro linear. Utilizou-se espaçamento de $1 \mathrm{~m}$ entrelinhas e $0,6 \mathrm{~m}$ entre plantas. A cada quinze dias a partir do transplante foram realizadas pulverizações com calda bordaleza, totalizando cinco aplicações. Também foi realizada uma capina manual trinta dias após o transplante.

Todas as cultivares possuem crescimento indeterminado. As cultivares Giovanna 1, Andréa, Bonnus 1, Débora, Thaty e Milenium são do tipo longa vida estrutural, enquanto que as cultivares Carmem e Stillus são longa vida rim e a cultivar Colibri é do tipo firme. Todas são resistentes às raça $1 \mathrm{e}$ 2 de Fusarium, exceto a Setcopa. Somente a cultivar Bônnus, Thaty e Setcopa não têm resistência a Verticilium, enquanto as cultivares Colibri, Carmem, Setcopa e Thaty não têm resistência a nematóides. As cultivares Colibri, Carmem e Stillus são resistentes ao mosaico e somente a Stillus é resistente ao geminivirus e a Bônus 1 ao Pseudomonas syringae (Alvarenga, 2004).

Após a colheita, os frutos foram encaminhados para o laboratório de Processamento de Produtos de Origem Vegetal da Escola de Agronomia da UFG, selecionados quanto à aparência, ausência de injúrias, podridões e grau de maturação. Quanto a esta última característica, os tomates foram separados em três estádios de maturação, identificados pelo desenvolvimento de cor da casca: (1) verde-rosado (pequena mudança 
de cor de verde para avermelhada na extremidade distal do fruto, ficando de 0 a $10 \%$ da superfície avermelhada ou amarelada); (2) rosado (entre 30 a $60 \%$ da superfície do fruto apresenta-se avermelhado ou róseo e verde-rosado, dependendo da cultivar) e (3) vermelho-claro (quando a superfície do fruto se encontra entre 60 a 90\% na coloração róseo-vermelha ou vermelha). Foram utilizados para a análise os frutos em estádio de maturação 3. Em seguida, foram lavados manualmente para remoção de impurezas superficiais, enxaguados em água corrente e submersos por 20 minutos em solução de hipoclorito de sódio a $100 \mu \mathrm{L} \mathrm{L}^{-1}$. Cada amostra foi composta por 20 frutos.

Para determinação de atributos físicos, utilizaram-se dez frutos de cada parcela, medindo-se o diâmetro transversal, o diâmetro longitudinal, a massa, o volume e a densidade. $\mathrm{O}$ diâmetro foi obtido por meio de paquímetro e a massa a partir de balança analítica. O volume foi estimado a partir do volume de água deslocado pela introdução de cada fruto em proveta de $500 \mathrm{~mL}$. A densidade foi calculada utilizando-se os dados de massa e volume obtidos (Machado \& Almeida, 1989).

Para a realização das análises físico-químicas, coletaram-se três frutos por parcela. Estes foram triturados em liquidificador e depois filtrados em papel de filtro qualitativo para determinação do $\mathrm{pH}$, do teor de sólidos solúveis (SS) e da acidez total (AT). O pH foi obtido com potenciômetro Análion PM 608; os SS com refratômetro Instrutherm RT-30ATC e a AT por titulação com solução NaOH 0,1 N. Todas as análises físico-químicas foram realizadas segundo as normas do Instituto Adolfo Lutz (2005). Calculou-se também a relação dos sólidos solúveis totais pela acidez total (SS/AT).

As dez cultivares de tomate produzidas no sistema orgânico, foram avaliadas quanto à preferência sensorial em relação à cor interna, cor externa, textura (consistência) e sabor, em um laboratório de análise sensorial, por cinqüenta provadores não treinados (consumidores). Aos provadores, foi apresentado em uma bandeja, um conjunto de fatias de cinco amostras, uma de cada cultivar, de forma aleatória balanceada, solicitando-se a indicação quanto à preferência relativa aos atributos cor externa, cor interna, textura e sabor. A soma das indicações para cada atributo foi calculada para cada cultivar, e foi elaborado um gráfico de barras para avaliação descritiva dos resultados (Meilgaard et al., 1999).

No segundo experimento, a cultivar Débora, produzida sob o sistema orgânico (safra 2010) na mesma propriedade e conduzida da mesma forma que no experimento um, foi comparada com a mesma cultivar manejada em sistema convencional, adquirida em feira livre (Pedra - Ceasa) da cidade de Goiânia-GO. Este tomate foi cultivado em Goianápolis-GO, sendo aplicado 2,5 t de calcário dolomítico por hectare, com 90 dias de antecedência em relação ao plantio. O preparo do solo foi realizado por meio de duas passagens de grade aradora no terreno. O transplante das mudas foi realizado no final de julho. Utilizou-se um stand de 17.000 mudas/ ha. A adubação, com formulação 04-14-08, foi parcelada em 5 vezes, totalizando $12,25 \mathrm{t} / \mathrm{ha}$. O manejo de plantas daninhas foi a capina manual aos 30 dias após o transplante. Utilizou-se o sistema de irrigação por sulcos. Utilizaram-se três a quatro aplicações de mistura de fungicidas e inseticidas por semana. A colheita iniciou-se no final de outubro.

A análise sensorial foi conduzida em uma feira livre em Goiânia, onde a cultivar de tomate Débora, produzida em sistema orgânico e no convencional, foi avaliada por noventa provadores não treinados, em relação aos atributos sensoriais. Utilizou-se o teste de aceitabilidade, onde se avaliaram os atributos aroma, cor, sabor e aparência geral, por meio da escala hedônica de nove pontos, que variou de 1 (desgostei extremamente) a 9 (gostei extremamente) (Stone \& Sidel, 2004). Uma fatia de cada amostra foi servida em bandeja acompanhada da ficha de avaliação. Nesta também, foi perguntada a idade dos provadores, se consumiam o produto em questão e o critério utilizado no momento da compra do produto.

No primeiro experimento, utilizou-se delineamento inteiramente ao acaso, com dez tratamentos (cultivar) e três repetições, totalizando 30 parcelas. Os dados físicos e químicos obtidos foram submetidos à análise de variância e as médias comparadas pelo teste Tukey a 5\% de probabilidade. Para avaliar a qualidade física e química dos frutos do segundo experimento foram utilizadas três amostras de cada sistema de produção estudado, cada uma composta por vinte frutos. Os dados obtidos foram determinados como descrito no experimento um e avaliados por meio de análise descritiva. Todas as análises físico-químicas foram realizadas em triplicata. Para a análise sensorial, utilizou-se delineamento de blocos ao acaso, onde se considerou que cada provador correspondia a um bloco (Stone \& Sidel, 2004).

\section{RESULTADOS E DISCUSSÃO}

A cultivar Setcopa apresentou a maior massa, que não diferiu $(p>0,05)$ da massa das cultivares Giovanna 1, Colibri, Milenium e Stillus, mas diferiu $(\mathrm{p} \leq 0,05)$ das demais (Tabela 1). A massa da cultivar Setcopa foi semelhante à verificada por Piñón (2011) em Cuba (casa de vegetação). As massas das cultivares Andréa e Débora foram as menores, não diferindo da massa das cultivares Colibri, Carmem, Tathy 1 e Bônus (Tabela 1). As massas obtidas no presente trabalho foram menores $(11,4-48,8 \%)$ que as referidas por Alvarenga (2004), que apresentou valores de massa entre 200-230 g para a cultivar Giovanna 1, 140-160 g para Colibri, 180-220 g para Carmem, 120-140 g para Andréa, 130150 g para Bonus-1, 230-240 g para Stillus e 130-140 g para Débora. Este autor não descreve em que condições os dados de massa de cada cultivar foram obtidos. Provavelmente, a diferença de massa dos frutos deve-se ao sistema de cultivo utilizado, provavelmente o convencional. A massa do tomate é muito importante sob o ponto de vista comercial, além de ser a melhor maneira de exprimir indiretamente o tamanho 
Tabela 1. Massa, volume, densidade, diâmetro longitudinal e diâmetro transversal de dez cultivares de "tomate de mesa", cultivados em sistema orgânico (mass, volume, density and longitudinal and transverse diameter of ten cultivars of tomato for fresh consumption, grown in organic system). Goiânia, UFG, 2009.

\begin{tabular}{lccccc}
\hline Cultivar & $\begin{array}{c}\text { Massa } \\
(\mathbf{g})^{\mathbf{1}}\end{array}$ & $\begin{array}{c}\text { Volume } \\
(\mathbf{m L})^{\mathbf{1}}\end{array}$ & $\begin{array}{c}\text { Densidade } \\
(\mathbf{g} / \mathbf{m L})^{\mathbf{1}}\end{array}$ & $\begin{array}{c}\text { Diâmetro } \\
\text { longitudinal } \\
\left(\mathbf{m m} \mathbf{1}^{\mathbf{1}}\right.\end{array}$ & $\begin{array}{c}\text { Diâmetro } \\
\text { transversal } \\
(\mathbf{m m})^{\mathbf{1}}\end{array}$ \\
\hline Giovanna 1 & $141,36^{\mathrm{ABC}}$ & $147,90^{\mathrm{A}}$ & $0,96^{\mathrm{AB}}$ & $63,80^{\mathrm{AB}}$ & $62,60^{\mathrm{BC}}$ \\
Colibri & $124,04^{\mathrm{ABCD}}$ & $133,10^{\mathrm{ABC}}$ & $0,94^{\mathrm{BC}}$ & $53,30^{\mathrm{E}}$ & $52,80^{\mathrm{E}}$ \\
Carmem & $112,57^{\mathrm{CD}}$ & $135,55^{\mathrm{AB}}$ & $0,84^{\mathrm{BC}}$ & $60,60^{\mathrm{BCD}}$ & $58,10^{\mathrm{CD}}$ \\
Andréa & $95,85^{\mathrm{D}}$ & $110,60^{\mathrm{BC}}$ & $0,86^{\mathrm{BC}}$ & $69,30^{\mathrm{A}}$ & $45,00^{\mathrm{F}}$ \\
Tathy 1 & $118,84^{\mathrm{BCD}}$ & $156,50^{\mathrm{A}}$ & $0,76^{\mathrm{C}}$ & $63,90^{\mathrm{AB}}$ & $62,30^{\mathrm{BC}}$ \\
Milenium & $146,85^{\mathrm{AB}}$ & $157,50^{\mathrm{A}}$ & $0,95^{\mathrm{BC}}$ & $53,10^{\mathrm{E}}$ & $69,80^{\mathrm{A}}$ \\
Bônus-1 & $114,31^{\mathrm{CD}}$ & $125,00^{\mathrm{ABC}}$ & $0,92^{\mathrm{BC}}$ & $51,20^{\mathrm{F}}$ & $53,90^{\mathrm{DE}}$ \\
Stillus & $141,11^{\mathrm{ABC}}$ & $136,00^{\mathrm{AB}}$ & $1,04^{\mathrm{AB}}$ & $64,50^{\mathrm{AB}}$ & $62,30^{\mathrm{BC}}$ \\
Débora & $98,00^{\mathrm{D}}$ & $99,00^{\mathrm{C}}$ & $0,99^{\mathrm{AB}}$ & $56,80^{\mathrm{CDE}}$ & $48,60^{\mathrm{EF}}$ \\
Setcopa & $153,20^{\mathrm{A}}$ & $136,00^{\mathrm{AB}}$ & $1,13^{\mathrm{A}}$ & $62,40^{\mathrm{BC}}$ & $64,40^{\mathrm{ABC}}$ \\
\hline
\end{tabular}

${ }^{1}$ Em uma mesma coluna, médias com letras iguais não diferem entre si pelo teste Tukey a 5\% de probabilidade (in column, averages with same letters do not differ amongst themselves for the Tukey test at $5 \%$ ).

Tabela 2. pH, sólidos solúveis, acidez total e relação entre sólidos solúveis e acidez total (SS/AT) de dez cultivares de "tomate de mesa", cultivados em sistema orgânico (pH, soluble solids, total acidity and relation soluble solids and total acidity (SS/TA) of ten cultivars of tomato, grown in organic system). Goiânia, UFG, 2009.

\begin{tabular}{llccc}
\hline Cultivar & $\mathbf{p H}^{\mathbf{1}}$ & $\begin{array}{c}\text { Sólidos solúveis } \\
\left({ }^{\circ} \mathbf{B r i x}\right)^{\mathbf{1}}\end{array}$ & $\begin{array}{c}\text { Acidez total } \\
\mathbf{( \% )} \mathbf{1}^{\mathbf{1 , 2}}\end{array}$ & $\mathbf{S S} / \mathbf{A T}^{\mathbf{1}}$ \\
\hline Giovanna 1 & $4,22^{\mathrm{D}}$ & $5,07^{\mathrm{B}}$ & $0,94^{\mathrm{A}}$ & $5,39^{\mathrm{C}}$ \\
Colibri & $4,38^{\mathrm{AB}}$ & $5,03^{\mathrm{BC}}$ & $0,57^{\mathrm{C}}$ & $8,82^{\mathrm{AB}}$ \\
Carmem & $4,27^{\mathrm{CD}}$ & $4,67^{\mathrm{C}}$ & $0,73^{\mathrm{B}}$ & $6,40^{\mathrm{BC}}$ \\
Andréa & $4,09^{\mathrm{E}}$ & $5,30^{\mathrm{AB}}$ & $0,58^{\mathrm{C}}$ & $9,14^{\mathrm{A}}$ \\
Tathy 1 & $4,27^{\mathrm{CD}}$ & $5,23^{\mathrm{AB}}$ & $0,58^{\mathrm{C}}$ & $9,02^{\mathrm{A}}$ \\
Milenium & $4,28^{\mathrm{CD}}$ & $5,60^{\mathrm{A}}$ & $0,74^{\mathrm{B}}$ & $7,57^{\mathrm{B}}$ \\
Bônus 1 & $4,36^{\mathrm{ABC}}$ & $5,00^{\mathrm{BC}}$ & $0,68^{\mathrm{BC}}$ & $7,35^{\mathrm{B}}$ \\
Stillus & $4,44^{\mathrm{A}}$ & $4,67^{\mathrm{C}}$ & $0,62^{\mathrm{BC}}$ & $7,53^{\mathrm{B}}$ \\
Débora & $4,28^{\mathrm{CD}}$ & $5,00^{\mathrm{BC}}$ & $0,59^{\mathrm{C}}$ & $8,48^{\mathrm{AB}}$ \\
Setcopa & $4,32^{\mathrm{BC}}$ & $5,00^{\mathrm{BC}}$ & $0,59^{\mathrm{C}}$ & $8,48^{\mathrm{AB}}$ \\
\hline
\end{tabular}

${ }^{1}$ Em uma mesma coluna, médias com letras iguais não diferem entre si pelo teste Tukey a $5 \%$ de probabilidade; ${ }^{2} \mathrm{~mL}$ de $\mathrm{NaOH} 0,1 \mathrm{~N} / 100 \mathrm{~g}$ amostra (in column, averages with same letters do not differ amongst themselves, Tukey test at 5\%).

do fruto.

As cultivares Millenium, Thaty $1 \mathrm{e}$ Giovanna 1 apresentaram os maiores volumes e diferiram significativamente $(p \leq 0,05)$ apenas das cultivares Andréa e Débora, que apresentaram o menor volume. A densidade dos frutos variou entre 0,76 e $1,13 \mathrm{~g} / \mathrm{mL}$, sendo que a cultivar Setcopa apresentou maior densidade, diferindo apenas das cultivares Tathy tando o rendimento, além de propiciar maior resistência contra amassamento durante as operações de pós-colheita, por possuírem forma mais compacta, com menor espaço interno de cavidades (Silva et al., 2000).

A cultivar Andréa não diferiu $(p>0,05)$ das cultivares Tathy 1 e Stillus, apresentando maiores diâmetros longitudinais, e diferindo das cultivares Giovanna 1, Colibri, Carmem, Milenium, Bônus-1, Débora e Setcopa (Tabela 1). Nas cultivares Giovanna 1, Colibri, Carmem, Andréa, Thaty 1, Stillus e Débora verificou-se formato oblongo (diâmetro longitudinal maior ao transversal). Os formatos apresentados pelas cultivares sob sistema orgânico no presente trabalho foram os mesmos que os cultivados sob sistema convencional (Alvarenga, 2004). Segundo produtores orgânicos com longa experiência na produção e comercialização de tomate, os frutos oblongos mais compridos geralmente são preferidos em detrimento aos redondos pelo consumidor. As demais cultivares (Setcopas, Bonus 1 e Milenium) apresentaram formato redondo.

De acordo com a classificação de tomate em função do diâmetro transversal (Brasil, 2002), nesta pesquisa, as cultivares Andrea, Débora, Bônus-1 e Setcopa foram classificadas como de calibre ou classe pequena, as cultivares Colibri, Carmem e Milenium como médias e as cultivares Giovanna 1, Thaty e Stillus como grandes. Produzindo frutos de tamanho reduzido, o tomaticultor tem dificuldade de colocar o seu produto no mercado de forma competitiva (Gualberto et al., 2002).

A composição dos tomates variou em função da cultivar. Em relação ao $\mathrm{pH}$, a cultivar Andréa apresentou o menor valor, diferindo significativamente $(p \leq 0,05)$ das demais, enquanto que a Stillus apresentou o maior $\mathrm{pH}$, não diferindo $(p>0,05)$ da Colibri e da Bônus 1 (Tabela 2). Valores de $\mathrm{pH}$ obtidos no presente estudo para as cultivares Carmem e Débora foram intermediários e similares aos valores obtidos por Borguini \& Silva (2005), também para as cultivares Carmem $(4,3)$ e Débora $(4,2)$ cultivadas em sistema orgânico. 
Valores de $\mathrm{pH}$ mais baixos se relacionam ao sabor mais ácido, além de colaborar para a conservação do fruto após a colheita. $\mathrm{O}$ balanço entre a acidez e o teor de sólidos solúveis na formação do sabor pode influenciar a aceitação e a preferência do consumidor.

Os teores de sólidos solúveis (SS) variaram entre 4,67 e $5,60^{\circ}$ Brix. Os maiores teores de SS foram observados nas cultivares Milenium, Andréia e Tathy 1. Estas cultivares não diferiram quanto ao teor de $\mathrm{SS}(\mathrm{p}>0,05)$ e foram seguidas pelas cultivares Giovanna 1, Colibri, Débora, Setcopa e Bonus 1, que também não diferiram entre si. As cultivares Carmem e Stillus apresentaram os menores teores de SS (Tabela 2).

Sólidos solúveis variando entre 4, 15 e $5,12^{\circ}$ Brix foram obtidos por Casa \& Evangelista (2009), na cultivar Epagri 19, cultivada em sistema orgânico. O teor de sólidos solúveis no fruto, além de ser uma característica genética, é influenciado pela adubação, temperatura e irrigação. Os sólidos solúveis, acumulam-se no final da fase de maturação, sendo constituídos por cerca de $65 \%$ de açúcares, e representam a percentagem (em peso) de sólidos que se encontram dissolvidos na água do alimento. No caso de frutas, os sólidos solúveis totais têm tendência a exibir maior concentração com a evolução da maturação, devido aos processos de biossíntese ou, ainda, pela degradação de polissacarídeos (Alvarenga, 2004; Borguini \& Silva, 2005).

A amplitude de variação da AT neste estudo foi de 0,57 a 0,94\% (Tabela 2). A cultivar Giovanna 1 apresentou maior AT, diferindo $(\mathrm{p} \leq 0,05)$ das demais cultivares. Enquanto que, menores AT foram verificadas nas cultivares Colibri, Andréa, Tathy 1, Débora e Setcopa, que não diferiram entre si $(p>0,05)$ e também não diferiram das cultivares Bônus-1 e Stillus. Acidez total (AT) no tomate indica a quantidade de ácidos orgânicos presentes e a adstringência do produto (Sampaio \& Fontes, 1998) e é a principal influência no sabor dos frutos. A AT em amostras de tomate da cultivar Santa Clara, cultivada em sistema orgânico, foi em torno de $0,20 \%$ (Ferreira et al.,

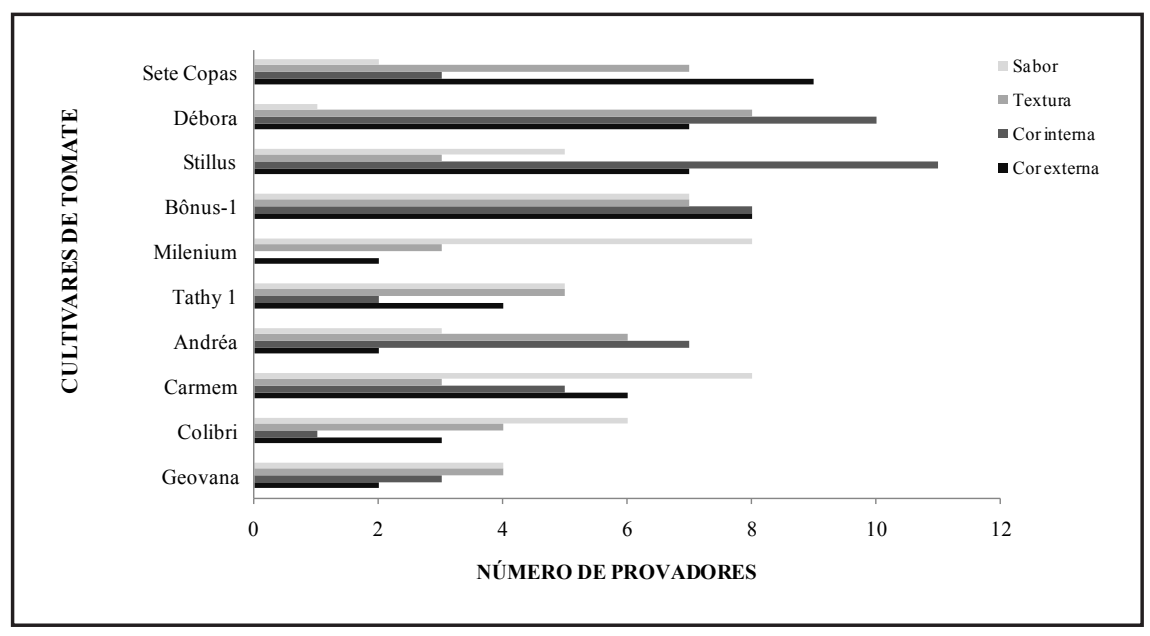

Figura 1. Preferência do consumidor entre dez cultivares de "tomate de mesa", cultivados em sistema orgânico, em relação à cor externa, cor interna, textura e sabor (consumer preference among ten cultivars of tomato for fresh consumption, cultivated in organic system, in relation to external color, internal color, texture and flavor). Goiânia, UFG, 2009.

Tabela 3. Características físicas e físico-químicas de tomate "de mesa" cultivar Débora, cultivado em sistema orgânico e convencional (physical and physicochemical characteristics of tomato for fresh consumption, cv. Deborah, grown under organic and conventional system). Goiânia, UFG, 2009.

\begin{tabular}{lcc}
\hline \multirow{2}{*}{ Características físicas } & \multicolumn{2}{c}{ Sistema de cultivo } \\
\cline { 2 - 3 } & Orgânico & Convencional \\
\hline Massa $(\mathrm{g})$ & 99,36 & 116,87 \\
Volume $(\mathrm{mL})$ & 98,50 & 116,50 \\
Densidade $\left(\mathrm{g} \mathrm{mL}^{-1}\right)$ & 1,01 & 1,01 \\
Diâmetro longitudinal (mm) & 81,10 & 81,50 \\
Diâmetro transversal (mm) & 50,80 & 48,80 \\
\hline Características físico-químicas & & \\
\hline pH & 4,91 & 4,47 \\
Sólidos solúveis ( $\left.{ }^{\circ} \mathrm{Brix}\right)$ & 4,48 & 3,93 \\
Acidez total (mL de NaOH 0,1N/100 g amostra) & 0,15 & 0,30 \\
SS/AT & 31,50 & 13,13 \\
\hline
\end{tabular}

2010b), inferior à AT obtida para todas as cultivares analisadas no presente trabalho.

No comércio de alimentos in natura, as preferências pelas cultivares mais doces ou mais ácidas dependem do hábito alimentar dos consumidores e dos padrões culturais da região. Portanto, é necessário disponibilizar ao mercado cultivares com diferentes padrões físico-químicos e sensoriais. Nesta pesquisa, as cultivares com maior teor de sólidos solúveis foram Milenium, Andrea e Tathy 1, enquanto a mais ácida foi a Giovana 1. Entretanto, o sabor do tomate está relacionado com a combinação de diversos constituintes químicos, desta- cando-se açúcares e ácidos. As cultivares Andréia e Tathy 1 apresentaram as maiores relações SS/AT, não diferindo ( $p>0,05)$ das cultivares Débora, Setcopa e Colibri. A cultivar Giovanna 1 apresentou a menor SS/AT, não diferindo da cultivar Carmem. Alto valor na relação indica uma excelente combinação de açúcar e ácido, a qual se correlaciona com sabor suave, enquanto que valores baixos estão diretamente relacionados com sabor ácido (Zambrano et al., 1996; Gil et al., 2002; Miguel et al., 2007). Wills \& Ku (2002) obtiveram para a cultivar Clarion, em sistema convencional, valores de SS/AT entre 3,2 e 3,8.

As cultivares Setcopa, Bônus-1, 
Stillus e Débora foram preferidas pelos provadores em relação à cor externa (Figura 1), pois apresentaram coloração vermelha mais intensa. Por outro lado, as cultivares Stillus, Débora, Bônus-1 e Andréa foram preferidas em relação à cor interna, devido à polpa e à placenta. Também apresentarem cor avermelhada mais intensa em relação às demais, que possuíam tons mais pálidos, ou mais esverdeados, ou mais amarelados, demonstrando que os genótipos mais ricos em licopeno são preferidos na região, maior produtora nacional de tomate industrial, cujos genótipos possuem obrigatoriamente esta característica e são comumente comercializados durante a safra, como tomate de mesa, refletindo esse hábito de consumo. Em relação à textura (mais macia) as cultivares escolhidas por maior número de provadores foram Débora, Setcopa, Bônus-1 e Andréa; enquanto que, para sabor (mais saborosa), as cultivares Carmem, Milenium, Bônus-1 e Colibri foram preferidas (Figura 1).

A cultivar Débora, produzida no sistema orgânico, foi selecionada dentre as demais, para ser comparada com a mesma cultivar conduzida em sistema convencional. Isso, pois, a cultivar Débora se apresentou entre as preferidas em relação aos atributos textura (maciez), cor externa e interna, além de apresentar a menor acidez, maior relação SS/AT e densidade elevada.

$\mathrm{Na}$ cultivar Débora, cultivada em sistema orgânico e convencional, o fruto convencional apresentou em média massa e volume 17,6 e 18,3\%, respectivamente, maior que o tomate orgânico, a densidade e os diâmetros longitudinais e transversais foram semelhantes (Tabela 3) e o formato do fruto em ambos os sistemas foi o oblongo. As diferenças entre a massa e o volume obtidas entre os dois sistemas, ou seja, do tamanho do fruto, está relacionado entre outros fatores à quantidade de água utilizada na rega, que também determinará maior ou menor concentração de componentes solúveis (Casquet, 1998).

Neste trabalho utilizaram-se diferentes sistemas de irrigação. $O$ tomate orgânico foi irrigado pelo sistema de gotejamento, enquanto que o convencional pelo de sulcos, cujo fornecimento de água é em quantidade bem superior. Outro aspecto provavelmente seja a quantidade de nutrientes fornecidos nos diferentes sistemas: no orgânico utilizou-se $2 \mathrm{t}$ de esterco de minhoca além de $500 \mathrm{~kg}$ de termofosfato Yorin, enquanto no convencional empregou-se 12,5 $\mathrm{t}$ do adubo formulado 04-12-08 por hectare. Isso provavelmente resultou em maior crescimento vegetativo das plantas, produção e translocação de substâncias orgânicas das folhas para os frutos e consequente obtenção de maior massa e volume dos tomates.

Ferreira et al. (2010a) compararam tomates convencionais e orgânicos oriundos de diferentes floradas e também obtiveram resultados de massa maiores para tomates convencionais $(161,40,160,82$ e $111,27 \mathrm{~g})$ que para orgânicos $(107,82,79,25$ e 88,08 g). Os resultados de densidade obtidos neste estudo estão em concordância com as densidades de tomate convencional $(0,96 \mathrm{~g} / \mathrm{mL})$ e orgânico (cultivar Santa Clara) $(0,94 \mathrm{~g} / \mathrm{mL})$ obtidas por Ferreira et al. (2010b).

$\mathrm{O}$ pH médio da amostra de tomate orgânico foi 9,8\% maior, o teor de SS $14 \%$ maior e a relação SS/AT 140\% maior, enquanto que a AT foi $50 \%$ menor que da amostra de tomate convencional (Tabela 3). As diferenças entre o pH, $\mathrm{SS}$, AT e SS/AT de amostras de tomate orgânico e convencional podem ser influenciadas pelas cultivares, estádios de maturação, manejo, fertilização, irrigação e composição do solo (Feltrin et al., 2002).

O tomate orgânico apresentou maior teor de SS que o tomate convencional, provavelmente devido ao maior teor de matéria seca. Pesquisa realizada por Schuphan (1974) na Alemanha, durante um período de 12 anos, visou a comparação entre dois padrões de aplicação de fertilizantes na produção de espinafre, batata, cenoura e repolho. Em um processo, foi utilizado um fertilizante convencional de alta solubilidade contendo NPK e no outro, adotado o uso de adubo orgânico. Os resultados revelaram que houve um decréscimo de $24 \%$ na produção quando se utilizou adubo orgânico. No entanto, com a aplicação da adubação orgânica, observou-se o acréscimo de matéria seca $(23 \%)$, proteína (18\%), vitamina C (28\%), açúcares totais $(19 \%)$.

Se o tomate orgânico fosse utilizado para o processamento de polpa de tomate, por exemplo, este teria um rendimento maior que o tomate convencional. Os teores de sólidos solúveis verificados no trabalho de Miguel et al. (2007) sobre tomate convencional, cultivar Débora, minimamente processado, variaram de 3,94 a $4,72^{\circ}$ Brix; esses resultados são 0,25 e 20,10\%, respectivamente, maiores que o do presente estudo. De forma geral, os estudos comparativos entre características físicas e químicas de diversos produtos hortícolas, cultivados em sistema orgânico e convencional, mostraram resultados variáveis (Darolt, 2003), necessitando de mais estudos.

Em estudo com tomates cultivados em sistema convencional, Raupp et al. (2009) obtiveram valores de $\mathrm{pH}$ de 4,2, 4,3, 4,2 e 4,1, para as cultivares Italiana, Débora Plus, Santa Cruz e Delícia, respectivamente. Esses resultados foram, respectivamente, $6,0,3,8,6,0$ e $8,3 \%$ menores que o obtido para a cultivar Débora produzida no sistema convencional do presente estudo.

Para a indústria de alimentos é melhor que o tomate possua maiores teores de SS e AT, mas, para o consumidor do tomate de mesa, a preferência pelas cultivares mais doces ou ácidas depende do hábito alimentar e dos padrões culturais da região. No estudo sobre a qualidade do tomate de mesa cultivado nos sistemas convencional e orgânico, Ferreira et al. (2010b) obtiveram AT para tomate convencional variando entre 0,21 e $0,58 \%$ e para tomate orgânico, entre 0,21 e $0,49 \%$. Esses resultados foram 40 e 226,67\% maiores que o obtido para o tomate orgânico do presente estudo. Já os resultados para o tomate convencional estão em concordância com os resultados dos autores citados anteriormente.

A diferença entre a relação SS/ATT para o tomate orgânico e convencional foi alta, sendo que o tomate orgânico 
apresentou o maior valor para essa relação. Segundo Gil et al. (2002), a relação SS/ATT ótima para o consumo de tomate é de 14,5. Assim, a cultivar Débora cultivada em sistema orgânico seria considerada ótima para o consumo, pois apresentou relação SS/ATT acima deste valor.

Os escores médios obtidos para o tomate cultivado no sistema orgânico e convencional foram, respectivamente, 7,49 e 7,06 para aroma, 7,14 e 7,31 para cor, 8,01 e 7,17 para sabor e 7,48 e 6,70 para aparência geral. Em relação à cor, a diferença entre os escores médios foi pequena $(2,3 \%)$ entre os tomates convencionais e orgânicos, mas quanto ao aroma, sabor e aparência geral, os escores médios variaram mais, entre $11,1-11,6 \%$, sendo os melhores escores atribuídos ao tomate orgânico. Os escores médios obtidos situaram-se entre gostei moderadamente e gostei muito, com exceção do sabor, que ficou entre gostei muito e gostei muitíssimo para o tomate orgânico; e aparência geral, que se localizou entre gostei pouco e gostei moderadamente para o tomate convencional. Com os dados obtidos foi possível inferir que tomate orgânico foi o mais aceito pelo grupo de provadores que participaram da análise sensorial. Borguini \& Silva (2005) avaliaram os atributos aroma, sabor, cor e aspecto geral em tomate orgânico e tomate convencional (ambos cultivar Débora) e apenas para o atributo sabor houve diferença significativa, ao nível de 5\%. Kader (2002) relata que o consumidor de frutas e hortaliças avalia, primeiramente, o aspecto geral deste e, posteriormente, a sua textura. Como o cliente não tem acesso à visualização do interior do fruto, usa somente a aparência geral no ato da compra ou sua experiência anterior para avaliar o produto.

Conclui-se, que mais se destacaram ao nível das necessidades do produtor (maior massa e densidade) as cultivares Setcopa, Milenium, Giovanna 1, Stillus, Thaty 1 e Colibri, enquanto que para o consumidor, cuja necessidade está mais voltada aos aspectos sensoriais e físico-químicos (cor, aroma, sabor, textura, sólidos solúveis e acidez), destacaram- -se as cultivares Débora, Bônus-1, Andréa, Setcopa, Stillus, Thaty 1 e Colibri. A cultivar Débora obteve menor massa, volume e diâmetro longitudinal quando cultivada em sistema orgânico, assim como maior teor de sólidos solúveis e menor acidez, que refletiu em maior aceitação quanto ao aroma, sabor e textura pelos consumidores.

\section{REFERÊNCIAS}

ALVARENGA MAR. 2004. Sistemas de produção em campo aberto e em ambiente protegido. In: ALVARENGA MAR (eds). Tomate: produção em campo, em casa de vegetação e em hidroponia. Editora UFLA, Lavras, 400p.

ARBOS KAP; FREITAS RJS; STERTZ SC; CARVALHO LA. 2010. Segurança alimentar de hortaliças orgânicas: aspectos sanitários e nutricionais. Ciência e Tecnologia de Alimentos 30: 215-220.

BORGUINI RG; SILVA MV. 2005. Características físico-químicas e sensoriais do tomate (Lycopersicon esculentum) produzido por cultivo orgânico em comparação ao convencional. Alimentos e Nutrição 16 : 355-361.

BRASIL. 2002. Ministério da Agricultura, Pecuária e Abastecimento. Portaria SARC $\mathrm{n}^{\circ} 085$ de 06 de março de 2002. Propõe o Regulamento técnico de identidade e qualidade para classificação do tomate. Diário Oficial da República Federativa do Brasil, Brasília.

CARDOSO MHWM; GOUVÊAAV; NÓBREGA AW; ABRANTES SMP. 2010. Validação de método para determinação de resíduos de agrotóxicos em tomate: uma experiência laboratorial. Ciência e Tecnologia de Alimentos 30: 63-72.

CASQUET E. 1998. Principios de economia agrária. Acribia, Zaragoza, 368 p.

CASA J; EVANGELISTA RM. 2009. Influência das épocas de colheita na qualidade de tomate cultivado em sistemas alternativos. Semina: Ciências Agrárias 30: 1101-1108.

DAROLT M. 2003. Comparação entre a qualidade do alimento orgânico e a do convencional. In: STRINGHETA PC; MUNIZ JN. (ed). Alimentos orgânicos: produção, tecnologia e certificação. Viçosa: UFV, p.289-31.

FAOSTAT. 2012. Database Results. Disponível em: <http://apps.fao.org>. Acesso em: 02 maio 2012.

FELTRIN DM; LOURENÇÃO AL; FURLANI PR; CARVALHO CRL. 2002. Efeitos de fontes de potássio na infestação de Bemisia Tabaci biótipo B e nas características de frutos de tomateiro sob ambiente protegido. Bragantia, 61: 49-57.

FERREIRA SMR; FREITAS RJS; KARKLE ENL; QUADROS DA; TULLIO LT; LIMA JJ. 2010a. Qualidade do tomate de mesa cultivado nos sistemas convencional e orgânico. Ciência e Tecnologia de Alimentos 30: 224-230.

FERREIRA SMR; QUADROS DA; KARKLE ENL; LIMA JJ; TULLIO LT; FREITAS RJS. 2010b. Qualidade pós-colheita do tomate de mesa convencional e orgânico. Ciência e Tecnologia de Alimentos 30: 858-864.

GUALBERTO R; BRAZ LT; BANZATTO DA. 2002. Produtividade, adaptabilidade e estabilidade fenotípica de cultivares de tomateiro sob diferentes condições de ambiente. Pesquisa Agropecuária Brasileira 37: 81-88.

GIL MI; CONESA MA; ARTÉS F. 2002. Quality changes in fresh cut tomato as affected by modified atmosphere packaging. Postharvest Biology and Technology 25: 199-207.

IAL - Instituto Adolfo Lutz. 2005. Normas analíticas do Instituto Adolfo Lutz: métodos fisico-químicos para análise de alimentos. Ministério da Saúde, Agência Nacional de Vigilância Sanitária, São Paulo, 1052p.

IBGE - Instituto Brasileiro de Geografia e Estatística. 2011. Levantamento sistemático da produção agrícola. 2011. Disponível em: $<$ http://www.ibge.gov.br/home/estatistica/ indicadores/agropecuaria/lspa/lspa_201108. pdf $>$. Acessado em: 20 out. 2011.

KADER AA. 2002. Postharvest technology of horticultural crops. Division of agriculture and natural resources publication, Los Angelis, $487 \mathrm{p}$.

MACHADO RCR; ALMEIDA HA. 1989. Estimativa do volume do fruto do cacau. Revista Brasileira de Fisiologia Vegetal 1: 115-117.

MELO PCT; TAMISO LG; AMBROSANO EJ; SCHAMMASS E; INOMOTO MM; SASAKI MEM; ROSSI F. 2009. Desempenho de cultivares de tomateiro em sistema orgânico sob cultivo protegido. Horticultura Brasileira 27: 553-559.

MEILGAARD M; CIVILLE GV; CARR BT. 1999. Senrory Evaluation Techniques, CRC Press, Inc., Boca Raton, FL, 387p.

MIGUEL ACA; DIAS JRPS; SPOTO MHF; RIZZO-BENATO RT. 2007. Qualidade de tomate "Débora" minimamente processado armazenado em dois tipos de embalagens. Horticultura Brasileira 25: 582-585.

PIÑÓN, M. 2011. Performance of tomato (Solanum lycopersicum) cultivars in a tropical sheltered production system. Ciencia e Investigación Agraria 38: 211-217.

RAUPP DS; GARDINGO JR; SCHEBESKI LS; AMADEU, CA; BORSATO AV. 2009. Processamento de tomate seco de diferentes cultivares. Acta Amazonica 39: 415-422.

SAMPAIO RA; FONTES PCR. 1998. Qualidade de frutos de tomateiro fertirrigado com potássio em solo coberto com polietileno preto. Horticultura Brasileira 16: 136-139.

SANJINEZ-ANGANDONA EJ; BRANCO IG; BITTENCOURT TU; MUNHOZ CL. 2011. Influência da geometria e da temperatura na cinética de secagem de tomate (Lycopersicum esculentum). Ciência e Tecnologia de Alimentos 31: 308-31. 
SCHUPHAN, W. 1974. Nutritive value of crops as influenced by organic and inorganic fertilizer treatment. Qualitas Plantarum: plant foods for human nutrition 23: 333-58.

SILVA JBC; GIORDANO LB; FURUMOTO O. 2000. Tomate para processamento industrial, Embrapa Hortaliças, Brasília, 168p.

SOUZA AS; BORGES SV; MAGALHÃES NF; RICARDO HV; AZEVEDO AD. 2008. Spraydried tomato powder: reconstitution properties and colour. Brazilian Archives of Biology and Technology 51: 807-814.

SOUZA JL; RESENDE PL. 2006. Manual de horticultura orgânica. 2. ed. Viçosa: Aprenda Fácil, 843 p.

STONE H; SIDEL JL. 2004. Sensory evaluation practices. San Diego: Elsevier, 658p.

TOLEDO DS; COSTA CA; BACCI L; FERNANDES LA; SOUZA MF. 2011.

Production and quality of tomato fruits under organic management. Horticultura Brasileira 29: 253-257.

WILLS RBH; KU VVV. 2002. Use of 1-MCP to extend the time to ripen of green tomatoes and postharvest life of ripe tomatoes. Postharvest Biology and Technology 26: 85-90.

ZAMBRANO J; MOYEJA J; PACHECO L. 1996. Efecto del estado de madurez en la composición y calidad de frutos de tomate. Agronomía Tropical 46: 61-72. 